PROCEEDINGS OF THE

AMERICAN MATHEMATICAL SOCIETY

Volume 136, Number 11, November 2008, Pages 3785-3793

S 0002-9939(08)09503-8

Article electronically published on May 20, 2008

\title{
NESTED SET COMPLEXES FOR POSETS AND THE BIER CONSTRUCTION
}

\author{
JULIANE LEHMANN
}

(Communicated by Paul Goerss)

\begin{abstract}
We generalize the concept of combinatorial nested set complexes to posets and exhibit the topological relationship between the arising nested set complexes and the order complex of the underlying poset. In particular, a sufficient condition is given so that this relationship is actually a subdivision.

We use the results to generalize the proof method of Cukić and Delucchi, so far restricted to semilattices, for a result of Björner, Paffenholz, Sjöstrand and Ziegler on the Bier construction on posets.
\end{abstract}

\section{INTRODUCTION}

Nested set complexes for semilattices were introduced by Feichtner and Kozlov in their paper [8] as a unifying framework for the study of De Concini-Procesi models of subspace arrangements and the resolution of singularities in toric varieties. Feichtner and Müller considered the topology of those complexes (9]); in particular, they prove that the nested set complex of any building set of a semilattice is homotopy equivalent to the order complex of the semilattice without its least element. These results found applications in the study of complexes of trees ([7]) and $k$-trees $([6])$.

The Bier construction was originally introduced by Thomas Bier in 1992 ([1]) as a construction on abstract simplicial complexes; more precisely, given an abstract simplicial complex $\mathcal{A}$, the deleted join of $\mathcal{A}$ with the combinatorial Alexander dual of $\mathcal{A}$ is another complex, the Bier sphere of $\mathcal{A}$. A short proof that this construction actually results in a sphere was given by de Longueville ([5]). In 2004, Björner, Paffenholz, Sjöstrand and Ziegler (2]) reinterpreted the construction in order-theoretic terms, by viewing an abstract simplicial complex as an ideal in a Boolean lattice. Then the corresponding Bier poset can be obtained as a subposet of the interval poset of the Boolean lattice, that is, the poset consisting of only those intervals that cross the ideal. This lends itself to an immediate generalization by considering arbitrary bounded posets instead of a Boolean lattice. It turned out that even in this general case the order complex of the Bier poset is a subdivision of the order complex of the original poset. The complexes that occur as intermediate steps of the subdivision are in general not order complexes, as remarked in [2].

Another view on the subject was taken by Čukić and Delucchi, who in 3 employed the theory of nested set complexes as a framework for the study of the Bier

Received by the editors September 19, 2007.

2000 Mathematics Subject Classification. Primary 06A07; Secondary 57Q05.

(C)2008 American Mathematical Society Reverts to public domain 28 years from publication 
construction. They found a new proof for the result of Björner et al. for semilattices, exhibiting the intermediate complexes as nested set complexes.

In this paper, we generalize the concept of nested sets to posets and exhibit the topological relationship between the arising nested set complexes and the order complex of the underlying poset. A sufficient condition is given so that this relationship is actually a subdivision. Using these results, we generalize the proof method of Čukić and Delucchi to posets and obtain a new proof for the result of Björner et al. in full generality, showing that the intermediate complexes are still nested set complexes.

This paper is organized as follows. The terminology used is given in Section 2. In Section 3, we extend the notion of nested set complexes to posets and prove our main Theorem 3.2 about the topological behaviour of nested set complexes under an extension of the building set. In particular, under certain circumstances, subdivisions take place, as is generally the case when considering semilattices. This allows us to apply the framework to the treatment of the Bier construction in Section 4 , in the same way as in $[3$, to obtain a new proof for the result of Björner et al. in full generality.

\section{Terminology}

In this paper, $P$ will denote a poset of finite length $(P$ will be used for the underlying set interchangeably). For a general reference on posets, see e.g. [4. We will here use the following terminology. Let $a, b \in P$ be elements of $P$; let $X$ be a subset of $P$. If $a \leq x$ for all $x \in X$, we write $a \leq X$; analogously $X \leq a$ means $x \leq a$ for all $x \in X$.

$P_{\leq X}$ denotes the set $\{a \in P: a \leq X\}$; analogously we write $P_{\geq X}$ for $\{a \in P$ : $a \geq X\}$. The set of upper bounds of $X$ is ub $X:=\min P_{\geq X}$; the set of lower bounds of $X$ is $\mathrm{lb} X:=\max P_{\leq X}$. If $P_{\geq X}$ has a least element $y$, so that ub $X=\{y\}$, then $y$ is called the join of $X$, denoted by $\bigvee_{x \in X} x$ or simply by $\bigvee X$. Conversely, if $\operatorname{lb} X=\{y\}$, then $y$ is called the meet of $X$, denoted by $\wedge X$. For $\bigvee\{a, b\}$ and $\bigwedge\{a, b\}$, the notation $a \vee b$ and $a \wedge b$ will be used, respectively.

We recall the difference between a poset and a (meet-)semilattice. In a semilattice $P$, for any finite subset $X$ of $P$, either the join of $X$ exists or ub $X$ is empty. But in a poset, sets of the form ub $X$ with $X \subset P, \mid$ ub $X \mid \geq 2$ can occur; these will be termed big cuts.

If $P$ has a least element, this will be denoted by $\hat{0}$; a greatest element will be denoted by $\hat{1}$. A bounded poset is a poset possessing $\hat{0}$ and $\hat{1} ; \bar{P}$ means $P \backslash\{\hat{0}, \hat{1}\}$. For elements $x \leq y$ of $P$, the interval $[x, y]$ is defined as the poset with elements $z \in P$ where $x \leq z \leq y$ and the order is induced by $P$. An ideal (order ideal or down-set) $I$ of $P$ is a subset of $P$ with the property that $x \in I$ and $y \leq x$ imply $y \in I$. In particular, if $P$ has a least element $\hat{0}$, then every ideal contains $\hat{0}$.

Now recall the definition of a building set of a poset, as given in 8 .

Definition 2.1. Let $P$ be a poset with $\hat{0}$, and let $G$ be a subset of $P_{>0}$. Denote by $F_{G}(x)$ the factors of $x$ in $G$, that is, $\max G_{\leq x}=\max \{g \in G: g \leq x\}$. Then $G$ is a building set of $P$ if for any $x \in P$ there is an isomorphism of posets

$$
\psi_{x}: \Pi_{i=1}^{t}\left[\hat{0}, x_{i}\right] \rightarrow[\hat{0}, x]
$$

satisfying $\psi_{x}\left(\left(\hat{0}, \ldots, \hat{0}, x_{i}, \hat{0}, \ldots, \hat{0}\right)\right)=x_{i}$ for all $i$, where $\left\{x_{1}, x_{2}, \ldots, x_{t}\right\}=F_{G}(x)$. 

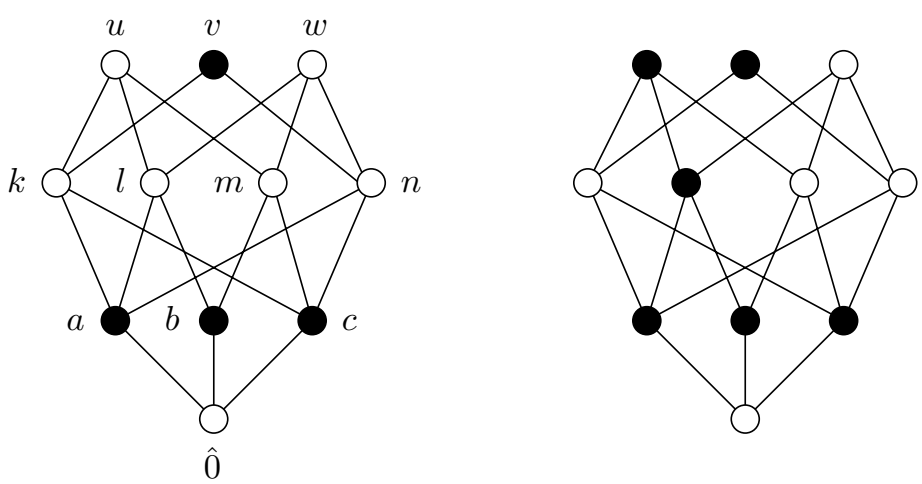

FiguRE 2.1. A poset with different building sets, marked in black.

Figure 2.1 shows a poset with different building sets.

For an abstract simplicial complex $\mathcal{A}$, the set of simplices of $\mathcal{A}$ will be denoted by $\mathcal{A}$ as well. In this paper, all abstract simplicial complexes are considered to contain the empty set. If $X$ is the subset of the vertex set of an abstract simplicial complex $\mathcal{A}$, then the subcomplex of $\mathcal{A}$ induced by $X$ has vertex set $X$ and set of simplices $\{\sigma \in \mathcal{A}: \sigma \subseteq X\}$.

In an abstract simplicial complex $\mathcal{A}$ with a face $\sigma$, the stellar subdivision of $\mathcal{A}$ at $\sigma$ is an abstract simplicial complex $\operatorname{sd}_{\mathcal{A}} \sigma$ with vertex set consisting of the vertex set of $\mathcal{A}$ and an additional vertex $\hat{\sigma}$, and with set of simplices defined as follows:

$$
\operatorname{sd}_{\mathcal{A}} \sigma:=\{\tau \in \mathcal{A}: \tau \nsupseteq \sigma\} \cup\{\tau \cup\{\hat{\sigma}\}: \tau \in \mathcal{A}, \tau \nsupseteq \sigma, \tau \cup \sigma \in \mathcal{A}\} .
$$

For a subcomplex $\tilde{\mathcal{A}}$ of $\mathcal{A}$, the cone over $\tilde{\mathcal{A}}$ is an abstract simplicial complex cone $\mathcal{A}_{\mathcal{A}} \tilde{\mathcal{A}}$ with vertex set again consisting of the vertex set of $\mathcal{A}$ and an additional vertex $a$, and with set of simplices defined as follows:

$$
\operatorname{cone}_{\mathcal{A}} \tilde{\mathcal{A}}:=\mathcal{A} \cup\{\tau \cup\{a\}: \tau \in \tilde{\mathcal{A}}\} .
$$

The order complex $\Delta(P)$ of $P$ is an abstract simplicial complex consisting of all chains of $P$.

\section{NeSted SET COMPLEXES AND THEIR BEHAVIOUR UNDER EXTENSION OF THE BUILDING SET}

The notion of a nested set introduced in [8] can be extended to posets as is.

Definition 3.1. Let $P$ be a poset of finite length with $\hat{0}$, and let $G$ be a building set of $P$. We call a finite subset $N \subset G$ nested if for every incomparable subset $A \subset N$ with $|A| \geq 2$ the join of $A$ exists and $\bigvee A \notin G$.

The nested sets in $G$ form an abstract simplicial complex, denoted $\mathcal{N}(P, G)$ (see Figure 3.1 for some examples). Note that every singleton subset of $G$ is nested in $G$; thus the vertices of $\mathcal{N}(P, G)$ correspond to the elements of $G$. Moreover, extending the building set has topological significance for the nested set complex.

Theorem 3.2. Let $P$ be a poset of finite length with $\hat{0}$, and let $G$ be a building set of $P$. Let $x \in \max P \backslash G$. Then $\tilde{G}:=G \cup\{x\}$ is a building set of $P$ and

(1) $\mathcal{N}(P, \tilde{G}) \cong \operatorname{sd}_{\mathcal{N}(P, G)} F_{G}(x)$ if $F_{G}(x)=\left\{x_{1}, \ldots, x_{t}\right\}$ is a face of $\mathcal{N}(P, G)$, 
(2) $\mathcal{N}(P, \tilde{G}) \cong$ cone $_{\mathcal{N}(P, G)} \mathcal{C}$ otherwise, where $\mathcal{C}$ is the subcomplex of $\mathcal{N}(P, G)$ induced by $C_{G}(x):=G_{<x} \cup G_{>x}$.

Proof. $\tilde{G}$ is clearly a building set of $P$.

Note that $F_{G}(x)$ is finite, since any interval in a poset of finite length cannot be isomorphic to an infinite product of non-trivial posets.

For (1), we need to see that

$$
\begin{aligned}
\mathcal{N}(P, \tilde{G})= & \left\{N \in \mathcal{N}(P, G): N \not \supset F_{G}(x)\right\} \\
& \cup\left\{N \cup\{x\}: N \in \mathcal{N}(P, G), N \not \supset F_{G}(x), N \cup F_{G}(x) \in \mathcal{N}(P, G)\right\} \\
\cong & \operatorname{sd}_{\mathcal{N}(P, G)} F_{G}(x) ;
\end{aligned}
$$

for (2), we need to see that

$$
\begin{aligned}
\mathcal{N}(P, \tilde{G}) & =\mathcal{N}(P, G) \cup\left\{N \cup\{x\}: N \in \mathcal{N}(P, G), N \subset C_{G}(x)\right\} \\
& \cong \operatorname{cone}_{\mathcal{N}(P, G)} \mathcal{C} .
\end{aligned}
$$

To this end, we show three equivalences:

$$
\mathcal{N}(P, G) \cap \mathcal{N}(P, \tilde{G})=\left\{N \in \mathcal{N}(P, G): N \not \supset F_{G}(x)\right\}=: \tilde{\mathcal{N}} .
$$

If $F_{G}(x) \in \mathcal{N}(P, G)$, then

$$
\mathcal{N}(P, \tilde{G}) \backslash \mathcal{N}(P, G)=\left\{N \cup\{x\}: N \in \tilde{\mathcal{N}}, N \cup F_{G}(x) \in \mathcal{N}(P, G)\right\} .
$$

If $F_{G}(x) \notin \mathcal{N}(P, G)$, then

$$
\mathcal{N}(P, \tilde{G}) \backslash \mathcal{N}(P, G)=\left\{N \cup\{x\}: N \in \mathcal{N}(P, G), N \subset C_{G}(x)\right\} .
$$

In Case 1, (a) and (b) give the result above; in Case 2, (a) and (c) are needed. Note that in Case 2 no nested set can contain $F_{G}(x)$, so $\tilde{\mathcal{N}}=\mathcal{N}(P, G)$ in that case.

In this proof let $\psi:=\psi_{x}$.

(a) Let $N$ be nested in $G$ and not nested in $\tilde{G}$. Then an incomparable subset $A=$ $\left\{a_{1}, \ldots, a_{s}\right\}$ of $N$ exists with $\bigvee A=x$, so $A \subset[\hat{0}, x]$. Let $a_{i}=\psi\left(a_{i 1}, a_{i 2}, \ldots, a_{i t}\right)$. Then since for all $a_{i}$ there exists $x_{j_{i}}$ such that $a_{i} \leq x_{j_{i}}$, we have $a_{i j}=\hat{0}$ for all $j \neq j_{i}$. So consider $A_{j}=\left\{a_{i} \in A: j_{i}=j\right\}$; since this is an incomparable subset of $A$ which is nested in $G$, the join of $A_{j}$ exists. Since the join of $A$ exists, it must coincide with the join of $\psi^{-1}(A)$ in $\Pi_{i}\left[\hat{0}, x_{i}\right]$, and the same for each $A_{j}$. Hence $\bigvee A_{j}=\psi\left(\bigvee \psi^{-1}\left(A_{j}\right)\right)=x_{j}$, which is in $G$. So $\left|A_{j}\right| \leq 1$ holds, and $A_{j}=\emptyset$ for any $j$ implies $\bigvee A \neq x$. Thus $A_{j}=\left\{x_{j}\right\}$ for all $j$, so $A=F_{G}(x) \subset N$.

Let $N$ be nested in $G$ and $F_{G}(x) \subset N . \quad F_{G}(x)$ is incomparable, and $\bigvee F_{G}(x)$ exists since $N$ is nested in $G$, so $\bigvee F_{G}(x)=\psi\left(\bigvee_{x_{i} \in F_{G}(x)} \psi^{-1}\left(x_{i}\right)\right)=$ $\psi\left(x_{1}, x_{2}, \ldots, x_{t}\right)=x$, which is not in $\tilde{G}$. Thus $N$ is not nested in $\tilde{G}$.

(b) Let $F_{G}(x)$ be nested in $G, N \subset \tilde{G}$ containing $x, N \backslash\{x\} \not \supset F_{G}(x)$ and $(N \backslash\{x\}) \cup F_{G}(x)$ be nested in $G$. Then $N$ is not nested in $G$ since $N \not \subset G$. Since $N \backslash\{x\}$ is nested in $G$ and does not contain $F_{G}(x), N \backslash\{x\}$ is nested in $\tilde{G}$ by (a). So only sets $A \subset N$ incomparable with $x \in A$ and $|A| \geq 2$ have to be investigated further. Note that for all $a \in A$ there is no $x_{i}$ with $a \leq x_{i}$, since otherwise $a \leq x$ follows in contradiction to $A$ being incomparable. Let $\overline{\tilde{A}}=(A \backslash\{x\}) \cup\left\{x_{i} \in F_{G}(x)\right.$ : $x_{i}$ is incomparable to all $\left.a \in A \backslash\{x\}\right\}$. Assume $|\tilde{A}|=1$, which means that $A=\{a, x\}$ and $a>x_{i}$ for all $x_{i}$; hence $a \geq \bigvee F_{G}(x)=x$, so $A$ is not incomparable. Hence $|\tilde{A}| \geq 2$, and since $\tilde{A} \subset(N \backslash\{x\}) \cup F_{G}(x)$ and $\tilde{A}$ is incomparable, $\bigvee \tilde{A}$ exists and is not in $G$. But $\bigvee \tilde{A} \geq F_{G}(x)$, so $\bigvee \tilde{A} \geq x$ with equality only if for all $a \in A$ there is 
$x_{i}$ with $x \geq a>x_{i}$, in contradiction to $\tilde{A} \subset G$. Hence $\bigvee \tilde{A}>x$, and therefore, by the choice of $x$, the join of $\tilde{A}$ is contained in $G$.

So there exists no $A \subset N$ incomparable with $x \in A$ and $|A| \geq 2$, so $N$ is nested in $\tilde{G}$.

Now let $F_{G}(x)$ be nested in $G$ and $N$ be nested in $\tilde{G}$ but not nested in $G$. As the existence of the join is independent of the considered building set, all nested sets in $\tilde{G}$ not containing $x$ are nested in $G$. So $x \in N$ and $N \backslash\{x\}$ is nested in $G$ and in $\tilde{G}$. Thus by (a), $N \backslash\{x\} \not \supset F_{G}(x)$.

Consider an incomparable subset $B$ of $(N \backslash\{x\}) \cup F_{G}(x)$, with $|B| \geq 2$, containing $x_{s} \in F_{G}(x) \backslash N$. Let $B_{N}=B \backslash F_{G}(x) \subset N \backslash\{x\}, B_{F}=B \cap F_{G}(x) \subset F_{G}(x)$. Since $F_{G}(x)$ is nested in $G, \bigvee B_{F}$ exists and is in $[\hat{0}, x] \backslash G$. If $B_{N}$ is empty, then $B=B_{F}$, and $\bigvee B \notin G$ exists.

So assume $\left|B_{N}\right| \geq 1$ in the following. As $N \supset B_{N} \cup\{x\}$ is nested in $\tilde{G}$, either $\bigvee B_{N} \cup\{x\} \notin \tilde{G}$ exists, which is not possible since $\bigvee B_{N} \cup\{x\} \geq x$, which by the choice of $x$ is in $\tilde{G}$, or $B_{N} \cup\{x\}$ is not incomparable. Assuming the existence of $b \in B_{N}$ with $b>x$ implies $b>x>x_{s}$, so $B$ would not have been incomparable. Let $\tilde{B}=\{x\} \cup\left\{b \in B_{N}: b\right.$ is incomparable to $\left.x\right\} ; \tilde{B} \subset N$ is incomparable. Assuming $|\tilde{B}| \geq 2$ we obtain a contradiction to $\bigvee \tilde{B} \notin \tilde{G}$ as above and thus to $N$ nested in $\tilde{G}$. Thus for all $b \in B_{N}$ there exists $x_{i}$ with $b<x_{i}$, so $B_{N} \subset[\hat{0}, x]$. Since $B$ is incomparable, these $x_{i}$ are not all in $B_{F}$, so there is w.l.o.g. a partition $\{[f],[t] \backslash[f]\}$ of $[t]$, where $[f]=\left\{i: x_{i} \in B_{F}\right\}$, and $\psi^{-1}\left(B_{N}\right) \subset \Pi_{i \in[f]}\{\hat{0}\} \times$ $\Pi_{i \in[t] \backslash[f]}\left[\hat{0}, x_{i}\right]$. So $\psi^{-1}\left(\bigvee B_{N}\right)=\left(\hat{0}, \ldots, \hat{0}, y_{f+1}, y_{f+2}, \ldots, y_{t}\right)$ and $\psi^{-1}\left(\bigvee B_{F}\right)=$ $\left(y_{1}, y_{2}, \ldots, y_{f}, \hat{0}, \ldots, \hat{0}\right)$ (both joins exist because $F_{G}(x), N \backslash\{x\}$ are nested in $G$ ), so $\bigvee B=\bigvee B_{F} \vee \bigvee B_{N}=\psi\left(y_{1}, y_{2}, \ldots, y_{t}\right)$ exists. Since $B_{F}, B_{N} \neq \emptyset$, at least two $y_{i} \neq \hat{0}$, so by definition of $F_{G}(x)$ the join of $B$ is not in $G$. Thus $(N \backslash\{x\}) \cup F_{G}(x)$ is nested in $G$.

(c) Let $F_{G}(x)$ not be nested in $G$ and $N \in \mathcal{N}(P, \tilde{G}) \backslash \mathcal{N}(P, G)$. As in (b), $x \in N$ and $N \backslash\{x\}$ is nested in $G$. Let $n \in N \backslash\{x\}$. If $\{n, x\} \subset N$ is incomparable, then $n \vee x$ exists and is not in $\tilde{G}$, which is impossible by the choice of $x$ since $n \vee x \geq x$. Thus $n \in C_{G}(x)$.

Let $F_{G}(x)$ not be nested in $G$, let $N \subset \tilde{G}$ with $x \in N$ and let $N \backslash\{x\}$ be a subset of $C_{G}(x)$ that is nested in $G$. Since $x \in N, N$ is not a subset of $G$, so is not nested in $G$. Let $A \subset N$ be incomparable with $|A| \geq 2$, so $A \subset N \backslash\{x\}$, so $\bigvee A \notin G$ exists. Hence by the choice of $x, A \subset G_{<x}$; that is, for all $a \in A$ there exists $x_{i} \in F_{G}(x)$ with $a \leq x_{i}$. As $x \in \mathrm{ub} F_{G}(x)$ (by isomorphism of $[\hat{0}, x]$ to the product of the intervals $\left.\left[\hat{0}, x_{i}\right]\right)$ and $F_{G}(x)$ is incomparable but not nested in $G, \bigvee F_{G}(x)$ does not exist, so there exists $y \in \mathrm{ub} F_{G}(x), y \neq x$. Since $y \geq A$, assuming $\bigvee A=x$ implies $y \geq x$, a contradiction to the choice of $y$. Hence $\bigvee A$ is not in $\tilde{G}=G \cup\{x\}$, so $N$ is nested in $\tilde{G}$.

Note that for the maximal building set $P_{>0}$ the nested set complex $\mathcal{N}\left(P, P_{>0 \hat{0}}\right)$ coincides with the order complex $\Delta\left(P_{>\hat{0}}\right)$ of $P_{>\hat{0}}$ by definition. Thus, by successively expanding a building set of $P$ as in the preceding theorem, we get a sequence of nested set complexes, eventually arriving at the order complex of the poset. Some steps of this process are shown in Figures 3.1 and 3.2. Now it turns out that by considering the big cuts of a poset, a guarantee can be given for some building sets to yield only stellar subdivisions in every expansion step. 

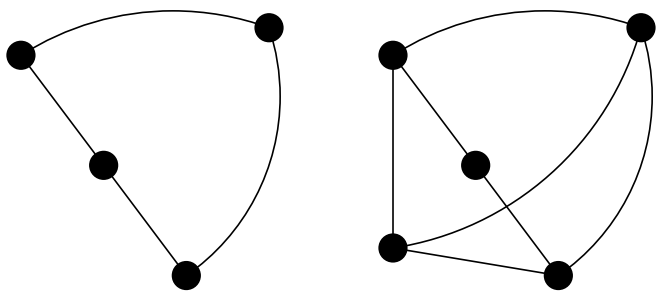

$\mathcal{N}\left(P, G_{1}\right)$

$\mathcal{N}\left(P, G_{2}\right)$
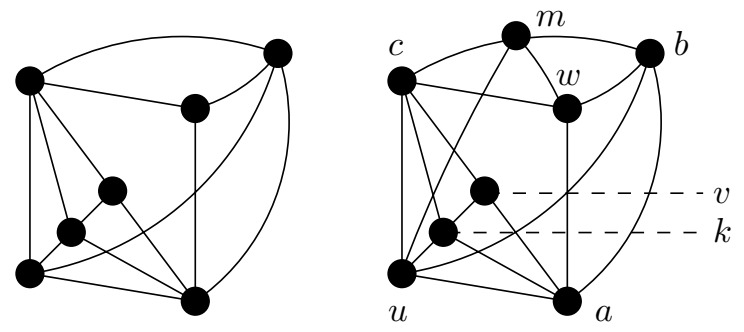

$\mathcal{N}\left(P, G_{3}\right)$

$\mathcal{N}\left(P, G_{4}\right)$

Figure 3.1. Nested set complexes of the poset $P$ shown in Figure 2.1. with the following building sets: $G_{1}=\{a, b, c, v\}, G_{2}=$ $\{a, b, c, v, u\}, G_{3}=\{a, b, c, v, u, w, k\}, G_{4}=\{a, b, c, v, u, w, k, m\}$. All triangles are part of the corresponding complex.

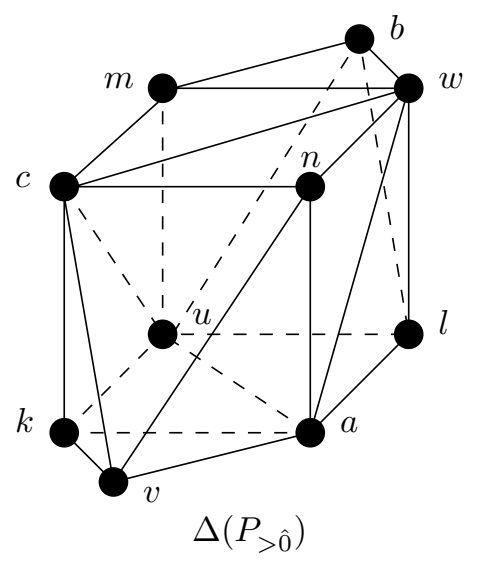

Figure 3.2. The reduced order complex of the poset $P$ shown in Figure 2.1, identical to the nested set complex for the building set $P_{>\hat{0}}$. Again, all triangles are part of the complex.

Lemma 3.3. If $G$ is a building set of $P$ with $\mathrm{ub} A \subseteq G$ for all $A \subseteq P$ with $|\mathrm{ub} A| \geq 2$, then $F_{G}(x) \in \mathcal{N}(P, G)$ for all $x \in P$.

Proof. Let $x \in P$; if $x \in G$, then $F_{G}(x)=\{x\}$ is nested in $G$. If $x \notin G$, then $F_{G}(x)=\left\{x_{1}, x_{2}, \ldots, x_{t}\right\}$; let $\psi:=\psi_{x}$. Let $A \subseteq F_{G}(x)$ with $|A| \geq 2$. Then $z=\psi\left(z_{1}, \ldots, z_{t}\right) \in \mathrm{ub} A$, where $z_{i}=x_{i}$ if $x_{i} \in A$ and $z_{i}=\hat{0}$ otherwise. Since 
we have $x_{i}<z \leq x$ for all $x_{i} \in A, z \notin G$ holds by the definition of $F_{G}(x)$. So ub $A=\{z\}$, or in other words, the join of $A$ exists (and equals $z$ ). Thus $F_{G}(x)$ is nested in $G$.

Theorem 3.4. For any building set $G$ of $P$ fulfilling the condition in Lemma 3.3, $\Delta\left(P_{>0 \hat{0}}\right)$ is a subdivision of $\mathcal{N}(P, G)$.

Proof. Stepwise expanding $G$ to the maximal building set $P_{>0}$ along a linear extension of $P_{>\hat{0}} \backslash G$ yields a stellar subdivision of the corresponding nested set complex in each step by Lemma 3.3 and Theorem 3.2. so $\mathcal{N}(P, G)$ subdivides to $\mathcal{N}\left(P, P_{>\hat{0}}\right)=\Delta\left(P_{>\hat{0}}\right)$.

\section{Applichtion to Bier posets}

The condition given in Lemma 3.3 is not at all necessary, as we will see now. The special structure of Bier posets allows us to determine easily that for a certain building set all extensions induce only stellar subdivisions of the corresponding nested set complexes, despite the occurrence of possibly many big cuts outside this building set.

But first of all we will recall the definition of a Bier poset.

Definition 4.1. Given a poset $P$ of finite length with $\hat{0}$ and a proper ideal $I$ of $P$, the $\operatorname{Bier}$ poset $\operatorname{Bier}(P, I)$ is a poset with

- elements $\{\hat{1}\} \cup\{[x, y]$ intervals of $P: x \in I, y \notin I\}$,

- order relation $[x, y] \leq[v, w]$ iff $x \leq v<w \leq y$ and $[x, y] \leq \hat{1}$ for all $I \ni x<$ $y \notin I$.

We now present a new structural proof using nested sets of the following result of Björner, Paffenholz, Sjöstrand and Ziegler in its full generality.

Theorem 4.2 ([2, Thm. 2.2]). Let $P$ be a bounded poset of finite length with a proper ideal I. Then the order complex of $\overline{\operatorname{Bier}(P, I)}$ is obtained from the order complex of $\bar{P}$ by stellar subdivision of the edges $\{x, y\}$ with $x<y, x \in I_{>0}, y \in \bar{P} \backslash I$ in order of increasing length of the corresponding intervals.

The argumentation follows [3], starting with a building set for $\operatorname{Bier}(P, I)_{<\hat{1}}$.

Proposition 4.3 ([3, Prop. 2.1]). For any bounded poset $P$ of finite length with proper ideal $I$,

$$
G=\left\{[x, \hat{1}],[\hat{0}, y]: x \in I_{>\hat{0}}, y \in \bar{P} \backslash I\right\}
$$

is a building set of $\operatorname{Bier}(P, I)_{<\hat{1}}$.

The proof of the above proposition in [3] does not use the lattice property or the finiteness and thus remains valid in the poset case. This building set of the Bier poset is very well behaved. Let $x \in I_{>0}, y \in \bar{P} \backslash I$, and let $[a, b] \in$ $\overline{\operatorname{Bier}(P, I)}_{\geq\{[x, \hat{1}],[\hat{0}, y]\}}$. Then we have $x \leq a<b \leq \hat{1}$ and $\hat{0} \leq a<b \leq y$, so $[x, y] \leq[a, b]$. Hence $[x, \hat{1}] \vee[\hat{0}, y]=[x, y]$.

This enables us to follow Cukić and Delucchi further; we find that their characterization of the nested sets in $G$ remains valid as well.

Lemma 4.4 ([3, Lem. 2.2]). With $G$ as above, a set $N \subset G$ is nested in $G$ iff it fulfills the following conditions:

(1) If $\left[\hat{0}, y_{1}\right],\left[\hat{0}, y_{2}\right] \in N$, then $y_{1}$ and $y_{2}$ are comparable. 
(2) If $\left[x_{1}, \hat{1}\right],\left[x_{2}, \hat{1}\right] \in N$, then $x_{1}$ and $x_{2}$ are comparable.

(3) If $[x, \hat{1}],[\hat{0}, y] \in N$, then $x<y$.

Proof. Let $N$ be nested in $G$. Assume that (1) is not true; that is, $\left[\hat{0}, y_{1}\right],\left[\hat{0}, y_{2}\right] \in N$ exist with $y_{1}, y_{2}$ incomparable. Then $\left[\hat{0}, y_{1}\right],\left[\hat{0}, y_{2}\right]$ are incomparable as well, so since $N$ is nested, $\left[\hat{0}, y_{1}\right] \vee\left[\hat{0}, y_{2}\right]$ exists and is not an element of $G$.

Let $S=\operatorname{lb}\left\{y_{1}, y_{2}\right\}$. If $p \in S \cap I$ exists, then for all $[a, b] \in \overline{\operatorname{Bier}(P, I)}_{\geq\left\{\left[\hat{0}, y_{1}\right],\left[\hat{0}, y_{2}\right]\right\}}$, we have $\hat{0} \leq a<b \leq y_{1}, y_{2}$; thus $b \leq p \in I$ holds, so $b \in I$, a contradiction to $[a, b] \in \operatorname{Bier}(P, I)$. Hence $\left[\hat{0}, y_{1}\right] \vee\left[\hat{0}, y_{2}\right]$ does not exist. So in the following let $S \cap I$ be empty.

Case 1: If $y_{1} \wedge y_{2}$ exists (and, as noted above, then is in $P \backslash I$ ), consider $[a, b] \in$ $\overline{\operatorname{Bier}(P, I)}_{\geq\left\{\left[\hat{0}, y_{1}\right],\left[\hat{0}, y_{2}\right]\right\}}$, which means $\hat{0} \leq a<b \leq y_{1}$ and $\hat{0} \leq a<b \leq y_{2}$, and thus $\left[\hat{0}, y_{1} \wedge y_{2}\right] \leq[a, b]$. Hence $\left[\hat{0}, y_{1}\right] \vee\left[\hat{0}, y_{2}\right]=\left[\hat{0}, y_{1} \wedge y_{2}\right] \in G$, a contradiction.

Case 2: If $y_{1} \wedge y_{2}$ does not exist, this means that $|S| \geq 2$. Observe that $[\hat{0}, s] \geq$ $\left[\hat{0}, y_{i}\right]$ for $i=1,2$ and all $s \in S$. Let $[p, q] \geq\left[\hat{0}, y_{i}\right]$ for $i=1,2$; that is, $\hat{0} \leq p<$ $q \leq y_{1}, y_{2}$. By definition of $S$, there exists $s \in S$ with $\hat{0} \leq p<q \leq s$, so we have $[p, q] \geq[\hat{0}, s]$. Thus, $[\hat{0}, s] \in \hat{S}:=\mathrm{ub}\left\{\left[\hat{0}, y_{1}\right],\left[\hat{0}, y_{2}\right]\right\}$ for all $s \in S$, so $|\hat{S}| \geq 2$ meaning that $\left[\hat{0}, y_{1}\right] \vee\left[\hat{0}, y_{2}\right]$ does not exist.

Analogously we obtain (2).

For $(3)$, we see that $[x, \hat{1}],[\hat{0}, y]$ are incomparable ( since $x \neq \hat{0}, y \neq \hat{1}$ ), so since $N$ is nested, $[x, \hat{1}] \vee[\hat{0}, y]=[p, q] \in \operatorname{Bier}(P, I)_{<\hat{1}}$. So $x \leq p<q \leq \hat{1}$ and $\hat{0} \leq p<q \leq y$ hold, implying $x<y$.

Conversely, consider a set $N \subset G$ fulfilling conditions (1)-(3). Let

$$
A=\left\{\left[x_{1}, \hat{1}\right], \ldots,\left[x_{s}, \hat{1}\right],\left[\hat{0}, y_{1}\right], \ldots,\left[\hat{0}, y_{t}\right]\right\}
$$

be an incomparable subset of $N$, with $|A| \geq 2$. By conditions (1) and (2), $s=t=1$, and as explained above, $\left[x_{1}, \hat{1}\right] \vee\left[\hat{0}, y_{1}\right]=\left[x_{1}, y_{1}\right] \notin G$ since $x_{1} \neq \hat{0}, y_{1} \neq \hat{1}$.

So the nested sets in this particular building set $G$ of a Bier poset coincide with those in the lattice case, which allows us to follow Čukić and Delucchi further, thus obtaining

Proposition 4.5 ([3, Prop. 2.3]). For $P, I$ and $G$ as above, $\mathcal{N}\left(\operatorname{Bier}(P, I)_{<\hat{1}}, G\right)=$ $\Delta(\bar{P})$.

Proof. As in 3], by Lemma 4.4 all nested sets $N$ in $G$ are of the form $N=$ $\left\{\left[x_{1}, \hat{1}\right], \ldots,\left[x_{s}, \hat{1}\right],\left[\hat{0}, y_{1}\right], \ldots,\left[\hat{0}, y_{t}\right]\right\}$, where $x_{1}<x_{2}<\ldots<x_{s}<y_{1}<y_{2}<\ldots<$ $y_{t}$, and $f: \mathcal{N}\left(\operatorname{Bier}(P, I)_{<\hat{1}}, G\right) \rightarrow \Delta(\bar{P})$, mapping a nested set to its underlying chain, is an inclusion-preserving bijection.

Now the good behavior of $G$ noted above comes into play once again. Considering any element $[x, y] \in \overline{\operatorname{Bier}(P, I)} \backslash G$, the factors of $[x, y]$ in $G$ are $F_{G}([x, y])=$ $\{[x, \hat{1}],[\hat{0}, y]\}$, which is an incomparable set with join $[x, y]$. Thus $F_{G}([x, y])$ is a nested set in $G$, but also in all building sets $\tilde{G}$ resulting from repeated application of Theorem 3.2 starting with the building set $G$. Hence in all applications of Theorem 3.2 only stellar subdivisions occur; more precisely we see that $\Delta(\overline{\operatorname{Bier}(P, I)}$ is obtained from $\mathcal{N}\left(\operatorname{Bier}(P, I)_{<\hat{1}}, G\right)=\Delta(\bar{P})$ by stellar subdivisions of all edges $f(\{[x, \hat{1}],[\hat{0}, y]\})=\{x<y\}$, in order of increasing length $\ell([x, y])$ in $P$ (since an interval of length 1 in $P$ is maximal in $\operatorname{Bier}(P, I)_{<\hat{1}}$, covering there the intervals 
of length 2 and so on). This concludes the proof of Theorem 4.2 via nested set complexes.

\section{ACKNOWLEDGEMENTS}

I would like to thank Dmitry Kozlov for introducing me to the problem and both him and Eva Maria Feichtner for helpful discussions.

\section{REFERENCES}

1. Thomas Bier, A remark on Alexander duality and the disjunct join, Preprint (1992).

2. Anders Björner, Andreas Paffenholz, Jonas Sjöstrand, and Günter M. Ziegler, Bier spheres and posets, Discrete Comput. Geom. 34 (2005), no. 1, 71-86. MR2140883 (2006k:06005)

3. Sonja Lj. Čukić and Emanuele Delucchi, Simplicial shellable spheres via combinatorial blowups, Proc. Amer. Math. Soc. 135 (2007), no. 8, 2403-2414 (electronic). MR2302561 (2008b:55027)

4. B. A. Davey and H. A. Priestley, Introduction to lattices and order, second ed., Cambridge University Press, New York, 2002. MR.1902334 (2003e:06001)

5. Mark de Longueville, Bier spheres and barycentric subdivision, J. Combin. Theory Ser. A 105 (2004), no. 2, 355-357. MR2046088 (2005d:52016)

6. Emanuele Delucchi, Subdivision of complexes of k-trees, Preprint (2005), available at arXiv:math/0509378v1.

7. Eva Maria Feichtner, Complexes of trees and nested set complexes, Pacific J. Math. 227 (2006), no. 2, 271-286. MR2263017 (2008a:05275)

8. Eva Maria Feichtner and Dmitry N. Kozlov, Incidence combinatorics of resolutions, Selecta Math. (N.S.) 10 (2004), no. 1, 37-60. MR2061222 (2006k:06008)

9. Eva Maria Feichtner and Irene Müller, On the topology of nested set complexes, Proc. Amer. Math. Soc. 133 (2005), no. 4, 999-1006 (electronic). MR2117200(2006c:06005)

Fachbereich Mathematik, Universität Bremen, 28359 Bremen, Germany

E-mail address: jlehmann@math.uni-bremen.de 